\title{
The rotation rates of massive stars ${ }^{\star}$ How slow are the slow ones?
}

\author{
J. O. Sundqvist ${ }^{1}$, S. Simón-Díaz ${ }^{2,3}$, J. Puls ${ }^{1}$, and N. Markova ${ }^{4}$ \\ 1 Universitätssternwarte München, Scheinerstr. 1, 81679 München, Germany \\ e-mail: mail@jonsundqvist.com \\ 2 Instituto de Astrofísica de Canarias, 38200 La Laguna, Tenerife, Spain \\ 3 Departamento de Astrofísica, Universidad de La Laguna, 38205 La Laguna, Tenerife, Spain \\ 4 Institute of Astronomy with NAO, BAS, PO Box 136, 4700 Smolyan, Bulgaria
}

Received 27 September 2013 / Accepted 14 October 2013

\begin{abstract}
Context. Rotation plays a key role in the life cycles of stars with masses above $\sim 8 M_{\odot}$. Hence, accurate knowledge of the rotation rates of such massive stars is critical for understanding their properties and for constraining models of their evolution.

Aims. This paper investigates the reliability of current methods used to derive projected rotation speeds $v \sin i$ from line-broadening signatures in the photospheric spectra of massive stars, focusing on stars that are not rapidly rotating.

Methods. We use slowly rotating magnetic O-stars with well-determined rotation periods to test the Fourier transform (FT) and goodness-of-fit (GOF) methods typically used to infer projected rotation rates of massive stars.

Results. For our two magnetic test stars with measured rotation periods longer than one year, i.e., with $v \sin i \lesssim 1 \mathrm{~km} \mathrm{~s}^{-1}$, we derive $v \sin i \approx 40-50 \mathrm{~km} \mathrm{~s}^{-1}$ from both the FT and GOF methods. These severe overestimates are most likely caused by an insufficient treatment of the competing broadening mechanisms referred to as microturbulence and macroturbulence.

Conclusions. These findings warn us not to rely uncritically on results from current standard techniques to derive projected rotation speeds of massive stars in the presence of significant additional line broadening, at least when $v \sin i \lesssim 50 \mathrm{~km} \mathrm{~s}^{-1}$. This may, for example, be crucial for i) determining the statistical distribution of observed rotation rates of massive stars; ii) interpreting the evolutionary status and spin-down histories of rotationally braked B-supergiants; and iii) explaining the deficiency of observed O-stars with spectroscopically inferred $v \sin i \approx 0 \mathrm{~km} \mathrm{~s}^{-1}$. Further investigations of potential shortcomings of the above techniques are presently under way.
\end{abstract}

Key words. stars: early-type - stars: rotation - stars: magnetic field - techniques: spectroscopic

\section{Introduction}

Rotation influences all phases of a massive star's life, from the initial collapse of the rotating molecular cloud (e.g., Bodenheimer 1995), over the star's main-sequence and evolved stages (e.g., Maeder \& Meynet 2000), to its violent death as a core-collapse supernova or hypernova, demises that in some cases may even produce very energetic bursts of gamma-rays (e.g., Yoon et al. 2006).

For most stars it is not possible to directly measure the rotation rate; instead, one typically infers the projected stellar rotation, $v \sin i$ (with inclination angle $i$ ), from the observed broadened line-spectrum. A key issue then becomes how to separate this rotational line broadening from other broadening components present in the atmosphere. In particular, it is well known that the occurrence of large so-called macroturbulence seriously complicates deriving accurate $v \sin i$ rates for massive stars that are not rotating too rapidly (Conti \& Ebbets 1977; Howarth et al. 1997; Ryans et al. 2002; Simón-Díaz \& Herrero 2007; Lefever et al. 2007; Simón-Díaz et al. 2010; Markova et al. 2011).

Several techniques for separating the effects of such "turbulent" and rotational broadening in massive stars have been

\footnotetext{
* Final reduced spectra are only available at the CDS via anonymous ftp to cdsarc.u-strasbg. fr (130.79.128.5) or via

http://cdsarc.u-strasbg.fr/viz-bin/qcat?J/A+A/559/L10
}

developed and applied (e.g., Ryans et al. 2002; Simón-Díaz \& Herrero 2007). For example, the Fourier transform (FT) method uses the first zero in the Fourier power spectrum to directly extract $v \sin i$, and the goodness-of-fit (GOF) method simply uses the different shapes of rotational and turbulent broadening to obtain a best fit of the spectral line. However, primarily because the underlying physics of additional line broadening in massive stars is still largely unknown (but see Aerts et al. 2009; Cantiello et al. 2009; Simón-Díaz et al. 2010; Shiode et al. 2013; Sundqvist et al. 2013), it is at present not clear to what degree these commonly used methods can actually isolate the rotational component, and so be used to derive quantitatively accurate values of $v \sin i$.

This Letter tests the FT and GOF methods by using highquality spectra of slowly rotating O-stars with detected largescale magnetic fields, collected within the Magnetism in Massive Stars project (MiMeS, Wade et al. 2012). The rotation period of these magnetic massive stars can be readily obtained from the observed variation of the longitudinal field (e.g., Borra \& Landstreet 1980; Bychkov et al. 2005) or photometric/spectral variations caused by their circumstellar magnetospheres (e.g., Landstreet \& Borra 1978; Howarth et al. 2007). In particular, we focus here on two magnetic O-stars with periods longer than one year, i.e., with $v \sin i \lesssim 1 \mathrm{~km} \mathrm{~s}^{-1}$. For these stars we derive projected rotation speeds using the FT and GOF methods, and 
critically evaluate the reliability of both techniques in the slowrotation regime of massive stars.

\section{Observations and method}

As our test objects, we use the Of?p-stars (Walborn 1972) HD 191612 and HD 108, the two known magnetic O-type stars with $v \sin i \lesssim 1 \mathrm{~km} \mathrm{~s}^{-1}$ and macroturbulent velocities in excess of $50 \mathrm{~km} \mathrm{~s}^{-1}$ (Martins et al. 2012; Sundqvist et al. 2013; Simón-Díaz \& Herrero 2013). Of these two stars, HD 191612 has the shorter rotation period, $P=538$ days, which along with its estimated stellar radius $14.5 R_{\odot}$ and inclination angle $i \approx 35-50^{\circ}$ yields $v \sin i<1 \mathrm{~km} \mathrm{~s}^{-1}$ (Howarth et al. 2007; Sundqvist et al. 2012). For the even longer period of HD 108, $v \sin i<1 \mathrm{~km} \mathrm{~s}^{-1}$ independent of $i$ (see, e.g., Petit et al. 2013). Although a few more magnetic O-stars with well constrained rotation periods are also known (see compilation by Petit et al. 2013), they all have either shorter periods or weaker macroturbulence. Shorter periods imply a non-zero and uncertain true projected rotation speed (due to uncertain stellar radii and even more so to an often poorly constrained inclination angle), and lower macroturbulence means a weaker influence of the primary competing broadening mechanism that complicates the derivation of accurate $v \sin i$ values. Since the aim of this paper is to test current methods using stars with known (and very slow) rotation rates, we restrict the analysis here to HD 191612 and HD 108, deferring to a future paper a complete study of all OB-stars with detected surface magnetic fields (see also discussion in Sect. 4).

High-quality spectra are retrieved from the extensive MiMeS database, and were obtained using the high-resolution $(R \sim$ $65000)$ spectropolarimeter ESPaDOnS at the Canada-France Hawaii Telescope (HD 191612) and its twin instrument Narval at the Télescope Bernard-Lyot (HD 108). As in Sundqvist et al. (2013), we focus on analyzing the stars' low states, defined according to when the cyclic $\mathrm{H} \alpha$ emission originating from their "dynamical magnetospheres" (Sundqvist et al. 2012) is at minimum. This should minimize any contamination of the photospheric lines by magnetospheric emission. However, for comparison, in Sect. 3 we also comment briefly on differences between this low state and the corresponding high state, defined by maximum $\mathrm{H} \alpha$ emission.

Since Zeeman splitting only contributes $\sim 1-2 \mathrm{~km} \mathrm{~s}^{-1}$ per kG in the optical, and the measured dipolar field strengths of HD 191612 and HD 108 are $2.5 \mathrm{kG}$ and $\sim 0.5 \mathrm{kG}$ (Wade et al. 2011; Martins et al. 2010), magnetic broadening is negligible for both stars (Sundqvist et al. 2013). This allows us to derive projected rotational velocities by means of the same techniques as for non-magnetic stars. For this objective, we use the photospheric O III $\lambda 5591$ line and apply the IACOB-BROAD tools developed by Simón-Díaz \& Herrero (2013). In summary, the FT method directly estimates $v \sin i$ from the position of the first zero in Fourier space, whereas the GOF method convolves synthetic line profiles for a range of projected rotational and macroturbulent velocities, creating a standard $\chi^{2}$-landscape from which a best combination of the two parameters is determined. For simplicity, in this letter we focus exclusively on the above mentioned O III line (as in Markova et al. 2013b and Simón-Díaz $\&$ Herrero 2013), but we have verified that a corresponding analysis of the C IV $\lambda 5801$ line gives very similar $v \sin i$ results (see also Howarth et al. 2007).

To be consistent with most recent studies of non-magnetic massive stars (e.g., Simón-Díaz et al. 2010; Najarro et al. 2011; Markova et al. 2013a), we adopt the "radial-tangential" (RT) formulation of Gaussian macroturbulence (rather than the isotropic
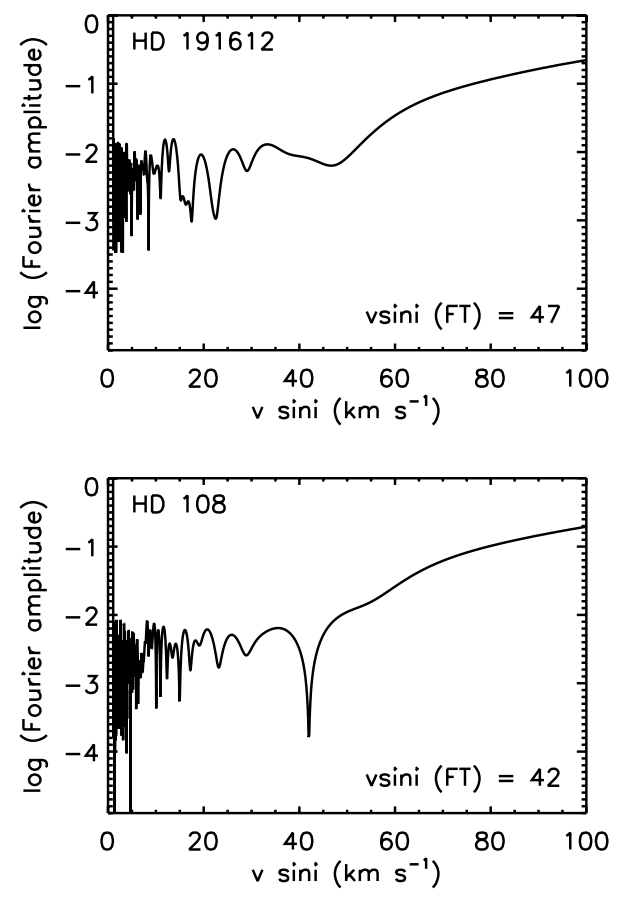

Fig. 1. Fourier power spectra of the O III $\lambda 5591$ line in HD 191612 and HD 108. In standard analysis, the position of the first minimum is used to infer the projected rotation speed of the star.

model used by Sundqvist et al. 2013). This RT model has been the primary choice in such previous work mainly because the FT and GOF methods then give consistent $v \sin i$ results (see Fig. 2, right panel). The model assumes that large-scale motions occur only radially and/or tangentially to the stellar surface (here we assume equal contributions in both directions), resulting in a more triangular-shaped profile (see Gray 2005) as compared to isotropic macroturbulence. Testing has shown that, for the same observed line profile, the different shapes of the RT and isotropic models result in characteristic macroturbulent velocities that differ on average by $\sim 20 \mathrm{~km} \mathrm{~s}^{-1}$ (with the RT model yielding systematically higher values, Markova et al. 2013b), and that the choice of macroturbulence also affects the derivation of $v \sin i$ (see Figs. 2 and 4, and discussion in Sect. 4).

\section{Results}

Figures 1 and 2 illustrate the derivation of the projected rotation speeds for HD 191612 and HD 108 using the FT and GOF techniques, respectively. The figures reveal how both methods yield $v \sin i \approx 40-50 \mathrm{~km} \mathrm{~s}^{-1}$, a severe overestimation compared to the $v \sin i \lesssim 1 \mathrm{~km} \mathrm{~s}^{-1}$ inferred from the variations of the longitudinal magnetic field (see Sects. 1 and 2) ${ }^{1}$.

The GOF contour-maps in Fig. 2 actually display quite wide ranges of allowed values for $v \sin i$ and macroturbulent velocities, where we note in particular that for HD $191612 v \sin i \approx 0 \mathrm{~km} \mathrm{~s}^{-1}$ cannot be ruled out with a $2 \sigma$ confidence, even though the best fit indicates a much higher value. However, from the first minima in the Fourier transforms displayed in Fig. 1 we clearly measure projected rotation speeds that deviate significantly from zero.

\footnotetext{
1 We note that while the analysis in this paper is based solely on spectra collected by the MiMeS collaboration, analysis of complementary IACOB spectra for HD 191612 and HD 108 (Simón-Díaz \& Herrero 2013) gives the same overestimated $v \sin i$ results as found here.
} 

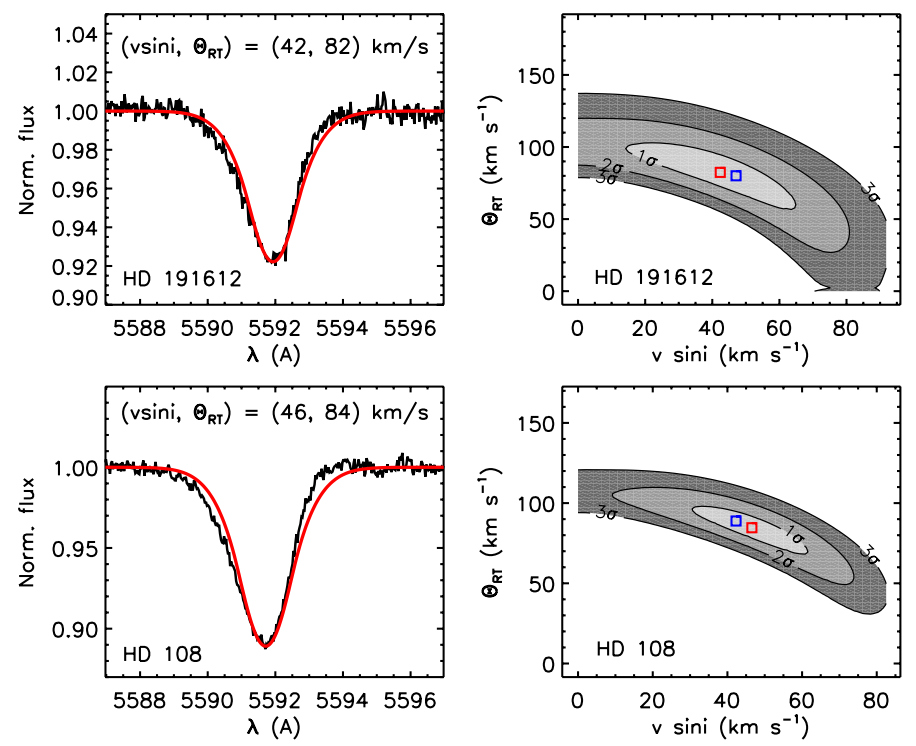

Fig. 2. Left: best fits of GOF analysis, with derived projected rotation speeds and characteristic macroturbulent velocities $\theta_{\mathrm{RT}}$ as indicated in the figure. Right: corresponding contour maps, with $1 \sigma, 2 \sigma$, and $3 \sigma$ confidence intervals indicated. The red squares indicate the best GOF and the blue squares the $v \sin i$ derived from the Fourier analysis in Fig. 1.

The line profiles displayed in Fig. 2 are asymmetric, which can influence the reliability of $v \sin i$ values, particularly when inferred from the FT method (e.g., Aerts et al. 2009). To test whether this asymmetry might be responsible for the overestimated projected rotation speeds, we artificially symmetrized the blue wings of the O III lines about their line-centers, and then re-analyzed the resulting symmetric profiles. However as illustrated for HD 191612 by Fig. 3, these symmetric profiles also give a clear high-velocity minimum in the Fourier power spectra, as well as a GOF contour-map similar to the one displayed in Fig. 2, resulting again in a best estimate $v \sin i \approx 40-50 \mathrm{~km} \mathrm{~s}^{-1}$. An equivalent analysis of HD 108, which is not shown in the figure, gives similar results, and symmetrizing the red wings of the profiles also yields highly overestimated projected rotation speeds, now on the order of $v \sin i \approx 30-40 \mathrm{~km} \mathrm{~s}^{-1}$ (due to the somewhat narrower redward wings in the original asymmetric profiles). To further test the possible impact of such line-profile asymmetry and variability, we also analyzed the O III line during HD 191612's high state (see Sect. 2), but that fitting yielded projected rotation speeds on the order of $40 \mathrm{~km} \mathrm{~s}^{-1}$ as well.

\section{Discussion and conclusions}

The central result of this paper is the $v \sin i \approx 40-50 \mathrm{~km} \mathrm{~s}^{-1}$ spectroscopically derived for two (magnetic) O-stars with measured rotation periods implying $v \sin i \lesssim 1 \mathrm{~km} \mathrm{~s}^{-1}$. Provided these results can be extrapolated to $\mathrm{O}$-stars in general (i.e., that there is no additional process that seriously contaminates the analysis here of the magnetic stars' photospheric line profiles), the largely overestimated $v \sin i$ values suggest that, in the presence of strong line broadening in addition to rotation, neither the FT nor the present implementation of the GOF method is able to derive reliable projected rotation speeds when $v \sin i \lesssim 50 \mathrm{~km} \mathrm{~s}^{-1}$. Ongoing (e.g., Simón-Díaz \& Herrero 2013) and future studies on larger samples will tell whether the stars examined here indeed are the forerunners of a more general problem.
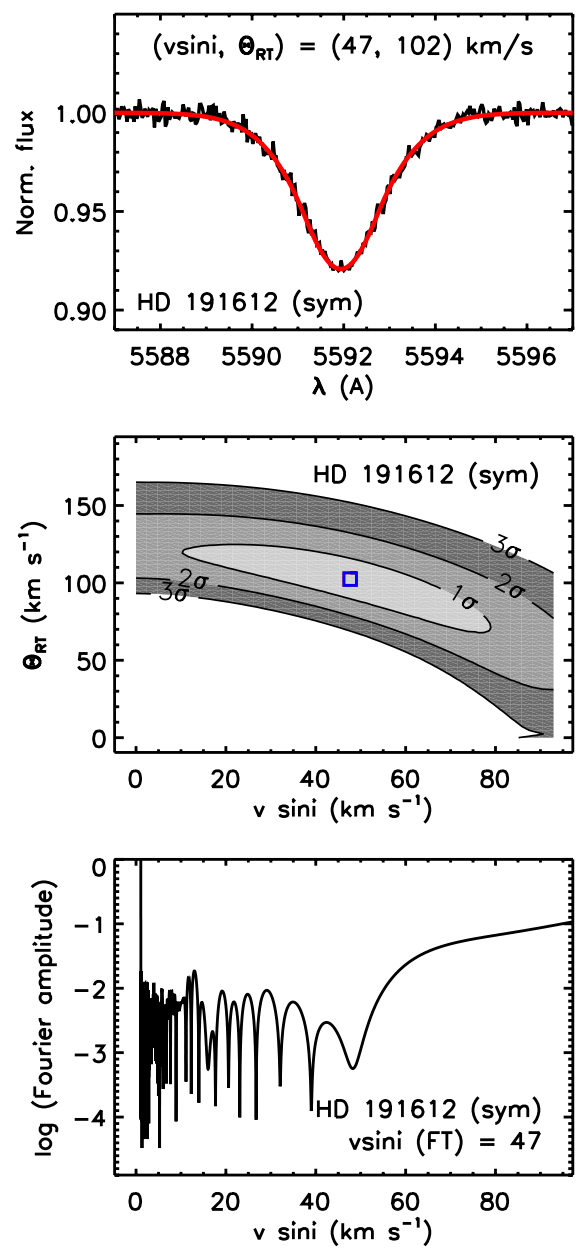

Fig. 3. Same as Figs. 1 and 2, but now with the observed O III line artificially symmetrized about line-center. We note the overlap of the blue and red squares in the middle panel, indicating perfect agreement between $v \sin i$ derived from the FT and GOF methods.

Such general shortcomings of the present-day techniques for inferring low projected rotation speeds may then have consequences for determining the statistical distribution of observed rotation rates of massive-star populations in the Galaxy and beyond (e.g., Hunter et al. 2008; Dufton et al. 2013; Ramírez-Agudelo et al. 2013; Simón-Díaz \& Herrero 2013). It may also help explain the apparent deficiency of observed $\mathrm{O}$ - and early B-stars with spectroscopically inferred $v \sin i \approx 0 \mathrm{~km} \mathrm{~s}^{-1}$, a long-standing problem (e.g., Penny 1996; Howarth et al. 1997) which only for the cases of late $\mathrm{O}$ - and B-dwarfs seems to have been alleviated by the consideration of additional broadening due to macroturbulence (Simón-Díaz \& Herrero 2007; Markova et al. 2013a,b; Simón-Díaz \& Herrero 2013). Furthermore, the results here may be important for interpreting the evolutionary status of massive B-supergiants, which are slow rotators, but which always seem to display rotation rates a bit higher than zero, on the order of $v \sin i \approx 30-40 \mathrm{~km} \mathrm{~s}^{-1}$ (Howarth et al. 1997; Markova et al. 2013a,b). If the rates of these Bsupergiants have also been overestimated, it would (at least for masses $M \gtrsim 35 M_{\odot}$, Markova et al. 2013a,b) bring observations in quantitative better agreement with current (single star) evolution models that predict these objects at essentially zero rotation speeds (Brott et al. 2011) $)^{2}$.

2 Resulting from strong wind-induced rotational braking when crossing to the cool side of the theoretical "bi-stability jump" of increased mass loss (Vink et al. 2010). 

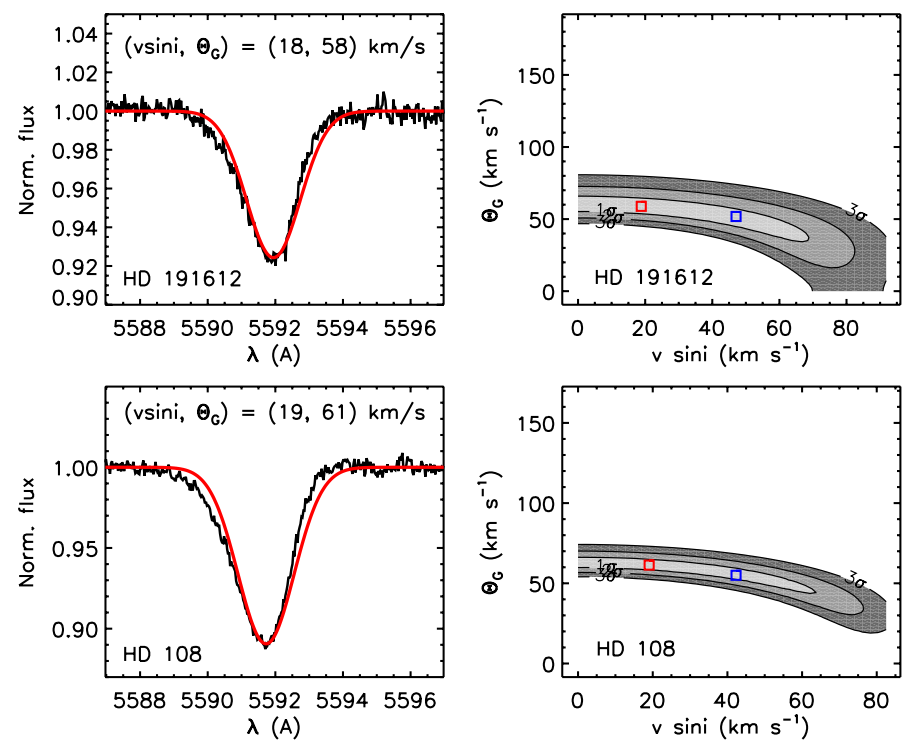

Fig. 4. As Fig. 2, but now assuming isotropic macroturbulence, with characteristic velocity $\theta_{\mathrm{G}}$, instead of radial-tangential (see Sect. 2). While a "best fit" is provided in the left panels, the solutions are in principle degenerate in the range $v \sin i \approx 0-60 \mathrm{~km} \mathrm{~s}^{-1}$, as illustrated by the contour maps in the right panels.

The overestimations of projected rotation speeds found for the magnetic stars here are most likely a result of insufficient treatments and physics-knowledge of the competing broadening mechanisms microturbulence and macroturbulence. For example, already Gray (1973) pointed out that classical atmospheric microturbulence can give Fourier-space minima in addition to those resulting from rotation; indeed, for relatively strong lines like those examined in this paper, tests using synthetic profiles show that microturbulent velocities of $\sim 20 \mathrm{~km} \mathrm{~s}^{-1}$ produce Fourier-space minima at frequencies corresponding to $\sim 30-40 \mathrm{~km} \mathrm{~s}^{-1}$ (Simón-Díaz \& Herrero 2013) ${ }^{3}$. Moreover, since (at least for lines with saturated cores) the effect of large microturbulence on the line shape can be mimicked by a suitable combination of rotation and macroturbulence (Simón-Díaz \& Herrero 2013), in these cases it is difficult to obtain accurate $v \sin i$ rates in the slow-rotation regime also when using the GOF method. Furthermore, the assumed shape of macroturbulence also affects the projected rotation speed inferred from a GOF solution (Simón-Díaz \& Herrero 2013). Indeed, repeating the GOF analysis in Sect. 3 but now assuming isotropic, instead of RT, macroturbulence results in degenerate solutions in the range $v \sin i \approx 0-60 \mathrm{~km} \mathrm{~s}^{-1}$, as illustrated by the contour maps in Fig. 4. This is consistent with Martins et al. (2012) and Sundqvist et al. (2013), who obtained reasonable line profile fits for HD 191612 and HD 108 when assuming zero projected rotation speeds and this type of isotropic macroturbulence. In summary, to make further progress it seems clear that a more realistic treatment of photospheric microturbulence and macroturbulence in massive stars is urgently needed.

Finally, this paper has focused on the extent to which reliable $v \sin i$ values of slowly rotating massive stars can be derived by current spectroscopic standard techniques, in the presence of a significant additional line-broadening component. However,

\footnotetext{
3 Microturbulent velocities of $\sim 20 \mathrm{~km} \mathrm{~s}^{-1}$ cannot be ruled out for O-type stars, particularly not for O-supergiants (e.g., Massey et al. 2013), but seem a bit high for the case of B-supergiants (e.g., Markova \& Puls 2008).
}

while for fast rotators the importance of such additional broadening decreases, there are other issues related to the derivation of accurate $v \sin i$ rates in that parameter range (like continuum normalization of shallow lines; see Simón-Díaz \& Herrero 2013). We thus plan to expand the study here to test the FT and GOF methods also for such faster rotators, using the significant number of relatively fast rotating magnetic B-stars with measured rotation periods (see Petit et al. 2013) present in the MiMeS database.

Acknowledgements. J.O.S. gratefully acknowledges support from DFG grant Pu117/8-1. The MiMeS project is thanked for providing the observed spectra of HD 108 and HD 191612. S.S.-D. acknowledges financial support from the Spanish Ministry of Economy and Competitiveness (MINECO) under the grants AYA2010-21697-C05-04, Consolider-Ingenio 2010 CSD2006-00070, and Severo Ochoa SEV-2011-0187, and by the Canary Islands Government under grant PID2010119.

\section{References}

Aerts, C., Puls, J., Godart, M., \& Dupret, M.-A. 2009, A\&A, 508, 409 Bodenheimer, P. 1995, ARA\&A, 33, 199

Borra, E. F., \& Landstreet, J. D. 1980, ApJS, 42, 421

Brott, I., de Mink, S. E., Cantiello, M., et al. 2011, A\&A, 530, A115 Bychkov, V. D., Bychkova, L. V., \& Madej, J. 2005, A\&A, 430, 1143 Cantiello, M., Langer, N., Brott, I., et al. 2009, A\&A, 499, 279

Conti, P. S., \& Ebbets, D. 1977, ApJ, 213, 438

Dufton, P. L., Langer, N., Dunstall, P. R., et al. 2013, A\&A, 550, A109

Gray, D. F. 1973, ApJ, 184, 461

Gray, D. F. 2005, The Observation and Analysis of Stellar Photospheres, 3rd edn. (Cambridge University Press)

Howarth, I. D., Siebert, K. W., Hussain, G. A. J., \& Prinja, R. K. 1997, MNRAS, 284,265

Howarth, I. D., Walborn, N. R., Lennon, D. J., et al. 2007, MNRAS, 381, 433

Hunter, I., Lennon, D. J., Dufton, P. L., et al. 2008, A\&A, 479, 541

Landstreet, J. D., \& Borra, E. F. 1978, ApJ, 224, L5

Lefever, K., Puls, J., \& Aerts, C. 2007, A\&A, 463, 1093

Maeder, A., \& Meynet, G. 2000, ARA\&A, 38, 143

Markova, N., \& Puls, J. 2008, A\&A, 478, 823

Markova, N., Markov, H., Puls, J., Simón-Díaz, S., \& Herrero, A. 2011, BgAJ, 17,54

Markova, N., Puls, J., Simón-Díaz, S., Herrero, A., \& Markov, H. 2013a, in Massive Stars: From alpha to Omega, http://alomega-conference .net Markova, N., Puls, J., Simón-Díaz, S., et al. 2013b, A\&A, accepted, DOI: $10.1051 / 0004-6361 / 201322661$

Martins, F., Donati, J.-F., Marcolino, W. L. F., et al. 2010, MNRAS, 407, 1423

Martins, F., Escolano, C., Wade, G. A., et al. 2012, A\&A, 538, A29

Massey, P., Neugent, K. F., Hillier, D. J., \& Puls, J. 2013, ApJ, 768, 6

Najarro, F., Hanson, M. M., \& Puls, J. 2011, A\&A, 535, A32

Penny, L. R. 1996, ApJ, 463, 737

Petit, V., Owocki, S. P., Wade, G. A., et al. 2013, MNRAS, 429, 398

Ramírez-Agudelo, O. H., Simón-Díaz, S., Sana, H., et al. 2013, A\&A, accepted DOI: $10.1051 / 0004-6361 / 201321986$

Ryans, R. S. I., Dufton, P. L., Rolleston, W. R. J., et al. 2002, MNRAS, 336, 577

Shiode, J. H., Quataert, E., Cantiello, M., \& Bildsten, L. 2013, MNRAS, 430, 1736

Simón-Díaz, S., \& Herrero, A. 2007, A\&A, 468, 1063

Simón-Díaz, S., \& Herrero 2013, A\&A, submitted

Simón-Díaz, S., Herrero, A., Uytterhoeven, K., et al. 2010, ApJ, 720, L174

Sundqvist, J. O., ud-Doula, A., Owocki, S. P., et al. 2012, MNRAS, 423, L21

Sundqvist, J. O., Petit, V., Owocki, S. P., et al. 2013, MNRAS, 433, 2497

Vink, J. S., Brott, I., Gräfener, G., et al. 2010, A\&A, 512, L7

Wade, G. A., Howarth, I. D., Townsend, R. H. D., et al. 2011, MNRAS, 416, 3160

Wade, G. A., Grunhut, J. H., \& MiMeS Collaboration 2012, in Circumstellar Dynamics at High Resolution, eds. A. C. Carciofi, \& T. Rivinius, ASP Conf. Ser., 464, 405

Walborn, N. R. 1972, AJ, 77, 312

Yoon, S., Langer, N., \& Norman, C. 2006, A\&A, 460, 199 